\title{
The Study of Ischemic Stroke
}

\author{
Yun Wang \\ Biomedicine and Pharmacy, University of Central Lancashire Preston, Lancashire PR1 1LJ, UK
}

\begin{abstract}
This study focuses on the pathology of ischemic stroke and the analysis of the disease. Ischemic stroke has become a serious disease that damages the health of the elderly year by year. Therefore, the continual analysis of ischemic stroke is able to make the disease be better prevented and effectively treated. This review contains diagnosis testing, treatment and prognoses, and analyzes the pros and cons of each method in each part. The prognostics treatment of patients is certainly an important part to be considered because most of patients are elderly. How to relieve the suffering of patients and help patients regain health are also the key points of this review. This review then records an interview of a patient who has the ischemic stroke and HIV. The interview mainly discusses the continuous treatment after left the hospital and the psychological. The patient affirmed some new treatments and also posed some psychological burden. In general, medical staff not only need to think of treating the patient's body, but also need to pay attention to the patient's psychological condition.
\end{abstract}

Key words: Ischemic stroke; Epidemiology; Atherosclerosis; Diagnostic test

Publication date: May, 2021; Publication online: 31 May, 2021

*Corresponding author: Yun Wang, yuness218@gmail.com

Stroke is a common cardia-cerebrovascular disease and $87 \%$ of strokes are ischemic (American Heart Association, 2020). Stroke is also a common complication of high blood pressure and heart disease.

\section{Reasons of ischemic stroke}

The underlying cause of ischemic stroke is insufficient blood supply for the brain. If the blood pressure is specifically slow or blood flow is too slow, the blood viscosity will increase or the blood coagulation will increase abnormally, eventually the thrombosis will occur. Cerebral vessels will become narrow in the circumstances, which will also lead to a blockage of cerebral blood flow and make brain tissue ischemia, hypoxia and softening, even necrosis, eventually cause cerebral vessels dysfunction and some clinical symptoms.

\section{Diagnostic testing}

There is a clinical method of identifying ischemic stroke named 'F.A.S.T.', which is mainly to observe whether the patient's face is drooping, or cannot raise his arms, or whether his language function is abnormal. A preliminary diagnosis can be made according to these three characteristics. In this case, the patient's wife exactly used 'F.A.S.T' method, before the patient did the MRI scan in the hospital to determine the disease. In addition to these two diagnostic methods, there are other tests can also be used for screening, such as carotid ultrasonography, coagulation test or cardiograph. These ways can be used to check the patient's blood and heart condition and are preferred screening before getting stroke. Since stroke is a complication, these screening methods can provide a sign of underlying risk to help make a preliminary judgment. Other useful methods such as Computed Tomography Angiography (CTA) and Digital Subtraction Angiography (DSA) are used to detect whether or not the cerebral blood vessels are abnormal. Although DSA has invasiveness and has a certain risk of inspection, it can help intuitively observe the stenosis of blood vessels and thus is the standard of diagnosis. CTA is one type of CT test and is the first choice for diagnosing the acute phase of the stroke. It can show the location and size of cerebral infarction, and it is three-dimensional imaging, which can be viewed from multiple angles. Therefore, CTA can make a diagnosis accurately and timely. In the detection of 
cardio-cerebrovascular disease, these two methods can be used jointly to complement each other if needed.

\section{Epidemiological determine}

Epidemiological research results show that the ischemic stroke has characteristics of high morbidity, high mortality and high disability. It is currently the second leading cause of death in the world (Mira, 2018). Stroke affects women more than men because women live longer than men, what is more, stroke typically occurs on women and recovery possibility is smaller. But for specific age, ischemic stroke is more common for people over forty years old, and male prevalence is greater than female. The occurrence of ischemic stroke is closely related to physical conditions. Heart problem will cause high blood pressure and damage the vascular system and finally lead the stroke. Weight level is also related to elevated blood cholesterol. Therefore, high blood pressure, heart disease, and adiposity are all the risk factors for getting ischemic stroke. These chronic diseases are likely to cause ischemic stroke. In addition, the patient is a smoker. According to the basis of extensive patient analysis on epidemiology, smokers are almost three times more likely to get stroke than non-smokers, thus smoking is another important risk factor. According to above discussion, the physical conditions and lifestyles of the patient in this case are highly consistent with mentioned risk factors of ischemic stroke. This shows that stroke has a high incidence of specific people (Mohan el at., 2009). These risk factors can be applied to people's daily initial self-diagnosis and reduce mortality as much as possible.

Ischemic stroke is mainly divided into two types, thrombotic and embolic. They are all caused by acute blockage of cerebral blood flow, but their etiology and pathogenesis are different.

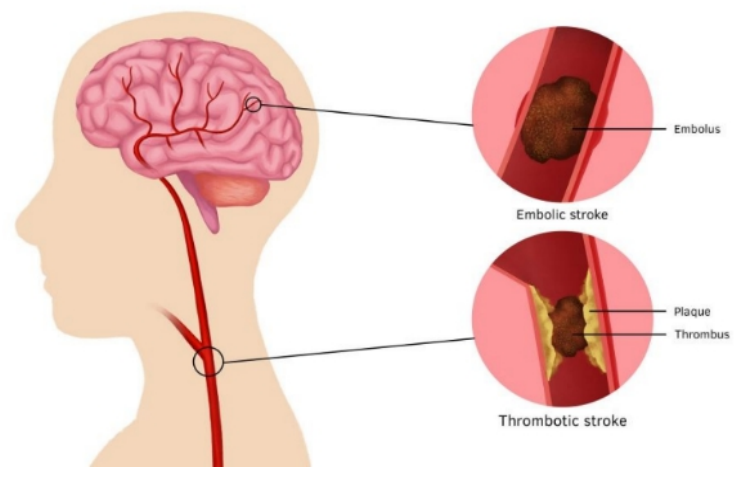

Figure 1. Thrombotic stroke and embolic stroke

Source: Color Atlas of Human Anatomy

As shown in Figure 1, the main cause of thrombotic stroke is blocked blood clot in brain. It occurs due to atherosclerosis and high blood viscosity and leads to cerebrovascular stenosis and local thrombosis, eventually blocks vessels and leads to ischemia and necrosis of brain tissue. Since the formation of blood clots is relatively long, the symptoms of thrombotic stroke cannot be observed timely until the clots formed, and it always happens during sleep or rest. Some patients have mild symptoms at first, then gradually worsen (Esherick, 2018). For embolic stroke, the source of emboli can be various and can be particles from elsewhere in the artery, such as air or fat. Because those emboli come from different places, only the original source is found during treatment can the disease be cured. Therefore, embolic stroke is much more dangerous and complex than thrombotic stroke. Since most emboli come from heart, embolic stroke mainly occurs on heart disease patient (Michael, 2013). Embolic stroke is the most acute type of stroke. By comparing the symptoms of these two types, aphasia and hemiplegia are more common for thrombotic stroke and epilepsy are more likely to appear in embolic stroke because of acuteness.

\section{Embolus}

The embolism disease can be divided into different types on the basis of embolus, which is the underlying cause of these two types of stroke. An embolus is an abnormal substance that is insoluble in the blood and can enter different places of body through blood flow. The emboli may be formed of solid, gas or liquid, but thrombosis is the most common one, such as thrombus.

\section{Thrombus \& Atherosclerosis}

Thrombus is formed on the basis of atherosclerosis. Atherosclerosis means the degeneration and necrosis of the arterial intima or the middle layer of the artery and is formed because some harmful factors, such as nicotine enter the bloodstream and damage the endovascular. Then some lipid substances enter the inner membrane through crevice and become plaque. These plaques break up and enter the bloodstream under the influence of some risk factors such as high blood pressure. The platelets will accumulate around the hole caused by a rupture and block it in the circumstances. Then more and more platelets bind with plaques and form thrombus (Duan, 1994). The formation of thrombus and atherosclerosis are inseparable, and atherosclerosis level is directly related to the occurrence of thrombotic stroke. Therefore, atherosclerosis is the most 
risky factor for thrombosis.

\section{Treatment methods}

Ischemic stroke is a chronic disease caused by many diseases and is prone to repeated attacks, thus it is very important to treat other diseases that can cause a stroke in evidence and to maintain a healthy lifestyle. For example, quit smoking, pay close attention to heart's health and eat less high-fat food. What is more, blood pressure, blood sugar, blood lipids and other risk factors for stroke should be measured frequently. However, dos and don'ts mentioned above can only play a supporting role and the most critical rehabilitation method is scientific treatment. Thrombosis and arteriosclerosis should be prevented and treated from the underlying cause. This method can improve the symptoms of ischemic stroke and can also reduce the high recurrence rate of stroke. If the disease occurs, it will be very important to give the patient anticoagulation treatment in time. Anticoagulation is to intervene in the coagulation process and reduce the further blockage of blood clots in the blood vessel. Anticoagulation can improve blood circulation and rescue cells and tissues in ischemic areas. Among all anticoagulants, hirudin is the strongest specific thrombin inhibitor, which can bind with the free thrombin in the plasma and clot to block the clotting process, instead of relying on clotting factors like other inhibitors (Sheffield, 2018). In addition its bleeding side effects are very small. Therefore, hirudin is very safe and reliable in the treatment of ischemic stroke. But currently the recombinant hirudin is not as effective as natural, thus it is necessary to continue study with hirudin in depth (Huhu, 2020).

\section{Prognoses}

After rescue and treatment, the biggest problem for patients who survived from ischemic stroke is recurrence. The stroke has a high recurrence rate, and the mortality and disability rate will increase after recurrence. Antihypertensive therapy is a useful method to reduce the possibility of recurrence, and is also the most important and most easily controlled risk factor for stroke (Rachmawati, 2016). The stroke will leave some sequelae after treatment, and the severity of the sequelae depends on the extent of the disease and whether the rescue is timely. If the disease is mild, the prognosis will be better. But for patients who relapse, the recovery will be slower. Sometimes severely ill patients will have a series of complications, which will affect the patient's prognosis. Physical disorder is one of the main sequelae, which manifested as hemiplegia. Some patients with mild symptoms can recover through rehabilitation training. After formal early rehabilitation training, $60 \%$ of stroke patients can take care of themselves (Morrison, 2014). Many patients also have severe language dysfunction. Under the circumstances, the psychological aspect of the patient should be focused. This is the case often ignored during treatment.

\section{Conclusion}

The purpose of treatment of ischemic stroke is to reduce the disability rate and improve the patient' quality of life. Because ischemic stroke is a high incidence of disease, it is very important to spread basic knowledge of diagnosis and treatment of stroke mentioned before, especially within families with elderly people at home. All the detection methods described above play their own unique role and some methods can even work together for better detection. For example, the carotid ultrasonography can be first used to determine the narrowing level of vessel, then the DSA can be used if needed. This combination test can maximize time savings and allow doctors to spot hidden dangers timely. Therefore, people are supposed to follow the principles of early prevention, early detection and early treatment.

\section{References}

[1] American Heart Association. (2020). 'Ischemic Stroke (Clots)'. Available at: https://www.stroke.org/en/about-stroke/types-of-stroke/ ischemic-stroke-clots. Accessed:19 November 2020.

[2] Duan, M. (1994). 'The molecular relationship between thrombus and atherosclerosis', Chinese Medical Journal, 2(4), PP.195-197.

[3] Esherick, J. S. (2018). Current Practice Guidelines in Primary Care 2018. New York, McGraw-Hill Education LLC.

[4] Guangwen, G. and Xu, W. (2016). Color Atlas of Human Anatomy. Bei Jing, People's Medical Publishing House.

[5] Huhu, H., Haitao, Z., Ru, W., Yi, Y., Xiaoyan, L., Yinye, W. and Yuanjun, Z. (2020). 'Improving long circulation and procoagulant platelet targeting by engineering of hirudin prodrug', Elsevier B.V, 589(1), PP.119869.

[6] Michael, K. (2013). Advanced BIOLOGY. Oxford, 
New York, Oxford University Press.

[7] Mira, K. and Andreas, L. (2018). 'Global Burden of Stroke', Thieme Medical Publishers, 38(2), PP.208-211.

[8] Mohan, K. M., Crichton, S. L., Grieve, A. P., Rudd, A. G., Wolfe, C. D. A. and Heuschman, P. U. (2009). 'Frequency and predictors for the risk of stroke recurrence up to 10 years after stroke: the South London Stroke Register', Journal of Neurology, Neurosurgery and Psychiatry, 80(9), PP. 1012-1018.

[9] Morrison, K. J. (2014). Fast facts for stroke care nursing: an expert guide in a nutshell. New York, Springer Publishing Company.
[10] Rachmawati, H. (2016). 'In Vitro Study On Antihypertensive and Antihypercholesterolemic Effects of Curcumin Nanoemulsion', Scientia Pharmacetica, 84(1), PP.131-140.

[11] Sheffield, W. P., Eltringham, S., Louise, J. and Bhakta, V. (2018). 'A factor Xla-ativatable hirudin-albumin fusion protein reduces thrombosis in mice without promoting blood loss', Springer Science and Business Media LLC, 18(1), PP.21.

[12] Yixian, Q. (2019). The knowledge list of advanced biology. Bei Jing, Beijing Normal University Publishing. 